\title{
Genetic variation based on microsatellite analysis of the oriental river prawn, Macrobrachium nipponense from Qiandao Lake in China
}

\author{
K.Y. Ma ${ }^{1}$, J.B. Feng ${ }^{1}$ and J.L. Li ${ }^{1,2}$ \\ ${ }^{1}$ Key Laboratory of Exploration and Utilization of Aquatic Genetic Resources, \\ Shanghai Ocean University, Ministry of Education, Shanghai, China \\ ${ }^{2}$ E-Institute of Shanghai Universities, Shanghai Ocean University, \\ Shanghai, China \\ Corresponding author: J.L. Li \\ E-mail: j11i2009@126.com
}

Genet. Mol. Res. 11 (4): 4235-4244 (2012)

Received February 1, 2012

Accepted June 20, 2012

Published September 20, 2012

DOI http://dx.doi.org/10.4238/2012.September.20.1

\begin{abstract}
The oriental river prawn (Macrobrachium nipponense) is an important freshwater prawn species in China. We collected 236 oriental river prawns from four wild stocks from Qiandao Lake, Zhejiang, China, and used nine polymorphic microsatellite markers to investigate their genetic diversity and structure, to facilitate the development of a selective breeding program. We found 185 alleles at nine loci in this sample. The observed heterozygosity $\left(H_{\mathrm{O}}\right)$ and expected heterozygosity $\left(H_{\mathrm{E}}\right)$ ranged from 0.43 to 0.89 and 0.56 to 0.95 , respectively. The four stocks of $M$. nipponense displayed high genetic diversity (14.33-15.89 alleles/locus, $H_{\mathrm{O}}=0.66-0.77$ and $\left.H_{\mathrm{E}}=0.78-0.88\right)$. Genetic diversity of the stock from Weiping town was lower than the stocks from the other locations. Mutation-drift equilibrium analysis showed no significant bottleneck effect. $F$-statistics among stocks ranged from 0.03 to 0.07 , indicating a moderate level of differentiation. Based on genetic structure analysis,
\end{abstract}


the 236 individuals from the four wild stocks could be divided into two potential populations. Overall, the 09CA, 09AY and 09JJ wild stocks had higher allelic and genetic diversity than the upstream 09WP stock. These three wild stocks could be used as founders for selective breeding.

Key words: Xin'anjiang Dam; Diversity; Population structure; Prawn

\section{INTRODUCTION}

The oriental river prawn, Macrobrachium nipponense, a member of the family Palaemonidae, is widely distributed in fresh waters in the low-salinity regions of estuaries in China and in most of Japan, Korea, Vietnam, and Myanmar (Yu and Miyake, 1972). M. nipponense is the most important commercial freshwater prawn in both China and Vietnam (Feng et al., 2008). The culture of $M$. nipponense in China began approximately 30 years ago, and the culture yields of this prawn exceeded 205,000 tons in 2008 (Ministry of Agriculture, China, 2009), accounting for the majority of its worldwide production. Growth in culture production has also gradually increased in recent years. M. nipponense is a relatively hardy species that adapts well to a wide range of physiological conditions. The females have a high fecundity and their larvae are easy to grow rapidly. The larvae reach a harvestable size in 6 to 12 months, depending on environmental conditions. In recent years, M. nipponense culture has been introduced in Singapore, the Philippines (Cai and Shokita, 2006), Iran (De Grave and Ghane, 2006), Uzbekistan (Mirabdullaev and Niyazov, 2005), and Iraq (Salman et al., 2006).

Qiandao Lake is on the west side of Zhejiang and the south side of Anhui Province. Originally, this lake was called Xin'anjiang Reservoir, and was a protected drinking water source. Qiandao Lake is now a large, artificial deep-water lake due to the construction of the Xin'anjiang Hydropower Station in 1959. Higher water and air temperatures in the water valley resulted from the establishment of the Xin'anjiang Reservoir. In addition, more mud has been deposited because of the reduced water flow. Moreover, decreases in natural runoff weakened the water purification and dilution functions. These changes were likely the main reasons for the reduced downstream biological diversity. M. nipponense is the main commercial freshwater crustacean in this lake.

Microsatellites are polymorphic loci present in nuclear DNA. They consist of repeated core sequences of $2 \sim 6$ bp in length. Microsatellite markers have been proven to be an efficient tool for evaluating the level of genetic variation in a species with limited genetic information available (Liu and Cordes, 2004; Yue et al., 2004, 2009; Liu et al., 2009) because of the high variability, abundance, neutrality, and co-dominance of microsatellite DNA. Polymorphic microsatellites have frequently been applied to genetic analysis and breeding programs of freshwater prawn populations (Wolfus et al., 1997; Chand et al., 2005; Chareontawee et al., 2007; Feng et al., 2008).

However, microsatellite analysis of the genetic diversity of $M$. nipponense is still in its early stages (Feng and Li, 2008; Feng et al., 2010; Ma et al., 2011). Moreover, the impact of water conservancy construction in Qiandao Lake on M. nipponense genetic diversity and structure has not been investigated. Whether the construction of the Xin'anjiang Hydropower Station has affected M. nipponense genetic diversity and structure is still unknown.

Here, we used nine microsatellite markers as a powerful tool to assess the genetic variation of wild M. nipponense stocks to establish a founder population for selective breeding pro- 
grams in Qiandao Lake. This genetic analysis could also provide basic scientific information for the protection and utilization of $M$. nipponense germplasm resources. Our analysis also serves as an assessment of the effect of water project construction on M. nipponense stocks.

\section{MATERIAL AND METHODS}

\section{Sample collection}

Stocks of M. nipponense were obtained from Jiangjia town (09JJ) (midstream of Qiandao Lake) and Chun'an town (09CA) (downstream of Qiandao Lake) in 2009. Subsequently, another two stocks were collected from Anyang town (09AY) (midstream of Qiandao Lake) and Weiping town (09WP) (upstream of Qiandao Lake). The specific locations of the sample collections are shown in Figure 1. From each wild stock, 59 individuals were randomly chosen. The tail muscle tissue of each individual was stored in $95 \%$ ethanol.

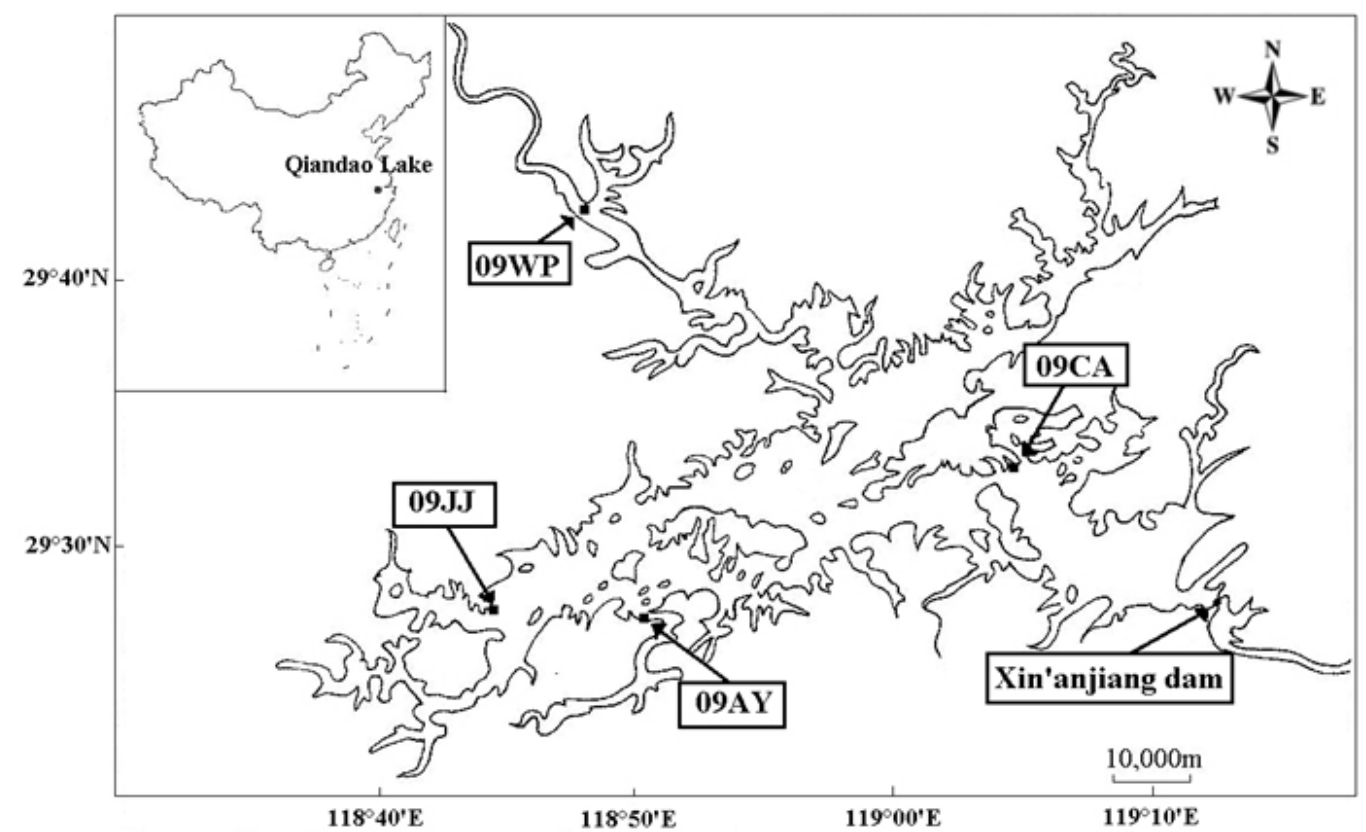

Figure 1. Four sampling locations of Macrobrachium nipponense stocks in Qiandao Lake. 09CA = wild stock of Chun'an town; 09AY = wild stock of Anyang town; 09JJ = wild stock of Jiangjia town; 09WP = wild stock of Weiping town.

\section{DNA extraction and microsatellite analysis}

DNA was isolated from the tail muscle tissue of 236 individuals selected from four wild stocks in Qiandao Lake. The phenol-chloroform method (Sambrook and Russell, 2001) was used to purify the DNA. The concentration and the purity of DNA were determined with a Nanodrop 2000c spectrophotometer (Thermo Scientific, USA). Genomic DNA samples were analyzed by $1 \%$ agarose gel electrophoresis and stored at $-20^{\circ} \mathrm{C}$. 
Nine previously isolated microsatellite markers in M. nipponense (Ma et al., 2010, 2011) were selected to genotype all samples. Polymerase chain reactions (PCR) were performed in 10$\mu \mathrm{L}$ volumes including: $0.25 \mathrm{U}$ Taq DNA polymerase (Tiangen, China), $1 \mathrm{X}$ Taq DNA polymerase PCR buffer (10 mM Tris-HCl, $1.5 \mathrm{mM} \mathrm{MgCl}, 50 \mathrm{mM} \mathrm{KCl}, \mathrm{pH} 8.3), 1 \mu \mathrm{M}$ of each primer, 200 $\mu \mathrm{M}$ dNTPs and $50 \mathrm{ng}$ template DNA. Samples were run on a PCR machine Mastercycler Pro $\mathrm{S}$ (Eppendorf, Germany) as follows: first, denaturation for $5 \mathrm{~min}$ at $94^{\circ} \mathrm{C}$, and then 30 cycles of $30 \mathrm{~s}$ at $94^{\circ} \mathrm{C}, 30 \mathrm{~s}$ at the annealing temperature and $30 \mathrm{~s}$ at $72^{\circ} \mathrm{C}$, followed by a final extension of $5 \mathrm{~min}$ at $72^{\circ} \mathrm{C}$. Primer pairs of the nine isolated microsatellites produced specific PCR products. Amplification products were resolved via the QIAxcel DNA High Resolution Kit (1200) (Qiagen, Germany), and pUC18 DNA/MspI (Tiangen) was used as a reference marker for allele size determination. Genotypes were exported to Excel tables for data analysis. Each microsatellite locus in the four stocks was examined for genotyping errors due to stuttering or large allele dropout and occurrence of null alleles using Microchecker (Van Oosterhout et al., 2004).

\section{Statistical analysis}

The Genepop 4.0 software (Rousset, 2008) was used to calculate the number of alleles $\left(N_{\mathrm{A}}\right)$, observed heterozygosity $\left(H_{\mathrm{O}}\right)$, expected heterozygosity $\left(H_{\mathrm{E}}\right)$, and HardyWeinberg equilibrium.

Pairwise genetic differentiation $F$-statistics $\left(F_{\mathrm{ST}}\right)$ and analysis of molecular variance (AMOVA) were determined using ARLEQUIN 3.1 (Excoffier et al., 2005). In AMOVA, the total variance was divided into variance among stocks, variance among individuals within stocks and variance within individuals. Nei's genetic distances were calculated using DISPAN (Nei et al., 1983). The relationships between the four stocks were then determined using the neighbor-joining (NJ) clustering tree based on a $D_{\mathrm{A}}$ genetic distance matrix.

The bottleneck hypothesis was tested under the two-phased model of mutation (TPM) using Bottleneck 1.2.02 (Cornuet and Luikart, 1996). For TPM, we used a model of $90 \%$ single-step mutations and the remaining 10\% multistep mutations and a variance of 10 . Based on the ratio of the number of individuals with heterozygosity excess and deficiency, the statistical significance of the analysis of dynamic changes was evaluated using the sign test and Wilcoxon sign rank test.

The genetic structure of different $M$. nipponense stocks was analyzed using Structure 2.3 (Rosenberg et al., 2002). In the assumption populations menu, we set the assumed $\mathrm{K}$ value from 1 to 4, with 10,000 repetitions of length of Burnin period and number of MCMC reps after Burnin.

\section{RESULTS}

\section{Genetic diversity in the four stocks}

Nine microsatellites were used to genotype the four stocks of M. nipponense. All of them were polymorphic and a total of 185 alleles were detected from all four stocks (Table 1). The $N_{\mathrm{A}}$ varied from 11 to 30 per locus. Locus Mni 82 was the most polymorphic with 30 alleles, whereas the microsatellite Mni86 had 11 alleles, which was the smallest number of alleles. Examination of genotyping errors using Microchecker revealed no evidence of large allele dropout or scoring error due to stuttering at any of the nine loci. Null alleles might have been present at some loci in each stock (e.g., Mni45, Mni76 and Mni93 loci). 


\begin{tabular}{|c|c|c|c|c|c|c|c|c|c|c|}
\hline \multirow[t]{2}{*}{ Stock } & \multicolumn{9}{|c|}{ Locus } & \multirow[t]{2}{*}{ Mean } \\
\hline & Mni30 & Mni34 & Mni45 & Mni52 & Mni76 & Mni82 & Mni86 & Mni93 & Mnilo2 & \\
\hline \multicolumn{11}{|l|}{$09 \mathrm{CA}$} \\
\hline$N_{\mathrm{A}}$ & 23 & 11 & 10 & 17 & 20 & 22 & 9 & 20 & 9 & 15.67 \\
\hline$H_{0}^{\mathrm{A}}$ & 0.73 & 0.72 & 0.71 & 0.72 & 0.76 & 0.85 & 0.76 & 0.77 & 0.27 & 0.70 \\
\hline $\begin{array}{c}H_{\mathrm{E}} \\
09 \mathrm{AY}\end{array}$ & $0.92 *$ & $0.86^{*}$ & $0.87 *$ & $0.84 *$ & $0.94 *$ & 0.95 & 0.80 & $0.93^{*}$ & $0.29^{*}$ & 0.82 \\
\hline$N_{\mathrm{a}}$ & 19 & 10 & 11 & 24 & 20 & 23 & 9 & 14 & 13 & 15.89 \\
\hline$H_{\mathrm{O}}^{\mathrm{A}}$ & 0.76 & 0.74 & 0.76 & 0.73 & 0.78 & 0.81 & 0.79 & 0.72 & 0.64 & 0.75 \\
\hline $\begin{array}{r}H_{\mathrm{E}} \\
09 \mathrm{JJ}\end{array}$ & $0.88^{*}$ & $0.85^{*}$ & $0.88^{*}$ & $0.92 *$ & $0.93 *$ & $0.94 *$ & $0.84 *$ & $0.85 *$ & $0.84 *$ & 0.88 \\
\hline$N_{\Delta}$ & 15 & 9 & 10 & 21 & 19 & 21 & 11 & 15 & 12 & 14.78 \\
\hline$H_{0}$ & 0.69 & 0.72 & 0.73 & 0.76 & 0.85 & 1.00 & 0.79 & 0.80 & 0.59 & 0.77 \\
\hline $\begin{array}{c}H_{\mathrm{E}}^{\circ} \\
09 \mathrm{WP}\end{array}$ & $0.85^{*}$ & $0.84 *$ & $0.87^{*}$ & $0.91 *$ & $0.93 *$ & $0.93 *$ & $0.86^{*}$ & $0.88^{*}$ & $0.77^{*}$ & 0.87 \\
\hline$N_{\mathrm{A}}$ & 15 & 13 & 11 & 21 & 14 & 22 & 9 & 12 & 12 & 14.33 \\
\hline$H_{\mathrm{O}}^{\mathrm{A}}$ & 0.38 & 0.69 & 0.66 & 0.76 & 0.79 & 0.90 & 0.74 & 0.65 & 0.37 & 0.66 \\
\hline $\begin{array}{c}H_{\mathrm{E}} \\
\text { Overall }\end{array}$ & $0.58 *$ & $0.87^{*}$ & $0.86^{*}$ & $0.93 *$ & $0.90 *$ & $0.93^{*}$ & $0.81 *$ & $0.73 *$ & $0.41^{*}$ & 0.78 \\
\hline$N_{\mathrm{A}}$ & 27 & 15 & 12 & 28 & 24 & 30 & 11 & 20 & 18 & - \\
\hline$H_{\mathrm{O}}^{\mathrm{A}}$ & 0.66 & 0.72 & 0.72 & 0.74 & 0.80 & 0.89 & 0.77 & 0.73 & 0.43 & - \\
\hline$H_{\mathrm{E}}$ & 0.86 & 0.86 & 0.88 & 0.92 & 0.94 & 0.95 & 0.86 & 0.89 & 0.56 & - \\
\hline
\end{tabular}

The 09AY stock had the largest average $N_{\mathrm{A}}(15.89)$ and the highest average $H_{\mathrm{E}}(0.88)$, while the 09WP stock had the smallest average $N_{\mathrm{A}}(14.33)$ and the lowest average $H_{\mathrm{E}}(0.78)$. The allele equilibrium status of the four stocks was tested for Hardy-Weinberg equilibrium (Table 1), and each locus significantly deviated from Hardy-Weinberg equilibrium, except for the Mni82 and Mni86 loci of the 09CA stock.

The results of the sign test and a Wilcoxon sign rank test are given in Table 2. According to both tests, no stock displayed significant heterozygosity excess $(\mathrm{P}>0.05)$. Analysis of stock bottleneck under the TPM revealed that the stocks might not have experienced a recent bottleneck $(\mathrm{P}>0.05)$.

Table 2. Departures from mutation-drift equilibrium in Macrobrachium nipponense stocks under two-phased model.

\begin{tabular}{lccc}
\hline Stock & He/Hd & \multicolumn{2}{c}{$\mathrm{P}$} \\
\cline { 3 - 4 } & & Sign test & Wilcoxon sign-rank \\
\hline 09CA & $6 / 3$ & 0.46 & 0.65 \\
09AY & $5 / 4$ & 0.53 & 0.57 \\
09J & $6 / 3$ & 0.46 & 0.42 \\
09WP & $5 / 4$ & 0.53 & 0.82 \\
\hline
\end{tabular}

09CA = wild stock of Chun'an town; 09AY = wild stock of Anyang town; 09JJ = wild stock of Jiangiia town; 09WP = wild stock of Weiping town. $\mathrm{He} / \mathrm{Hd}=$ ratio of number of individuals with a heterozygosity excess to the number with heterozygosity deficiency. $\mathrm{P}$ = possibility of heterozygosity excess.

\section{Genetic differentiation and relationships among populations}

Pairwise genetic differentiations based on allele frequency were determined for the 
four wild stocks of M. nipponense (Table 3). The analysis of pairwise genetic differentiation revealed that the $F_{\mathrm{ST}}$ values ranged from 0.03 to 0.07 , which was in line with a moderate level of genetic differentiation (Hartl and Clark, 1997). The $F_{\mathrm{ST}}$ value between the 09CA and 09JJ stocks was the lowest $\left(F_{\mathrm{ST}}=0.03\right)$; however, the highest $F_{\mathrm{ST}}$ value was found between the 09AY and 09WP stocks $\left(F_{\mathrm{ST}}=0.07\right)$. The $F_{\mathrm{ST}}$ value between the 09WP stock and the other three stocks displayed a significant difference $(\mathrm{P}<0.05)$.

\begin{tabular}{|c|c|c|c|}
\hline Stock & $09 \mathrm{CA}$ & 09AY & 09JJ \\
\hline 09AY & 0.05 & & \\
\hline 09JJ & 0.03 & 0.03 & \\
\hline $09 \mathrm{WP}$ & $0.06^{* *}$ & $0.07 *$ & $0.05^{*}$ \\
\hline
\end{tabular}

09CA = wild stock of Chun' an town; 09AY = wild stock of Anyang town; 09JJ = wild stock of Jiangiia town; 09WP $=$ wild stock of Weiping town. ${ }^{*} F_{\mathrm{ST}}$ were significant $(\mathrm{P}<0.05) ; * * F_{\mathrm{ST}}$ were extremely significant $(\mathrm{P}<0.01)$.

AMOVA revealed that the genetic variation within individuals was $95.87 \%$, and the genetic variation between stocks was $1.16 \%(\mathrm{P}<0.05)$ (Table 4$)$, which suggested a moderate level of genetic differentiation as well.

Table 4. AMOVA analysis among Macrobrachium nipponense stocks.

\begin{tabular}{lrccr}
\hline Source of variation & d.f. & Sum of squares & Variance components & Percentage of variance \\
\hline Among stocks & 3 & 3.27 & 0.01 & $1.16 \% *$ \\
Among individuals within stocks & 232 & 107.52 & 0.01 & $2.96 \%$ \\
Within individuals & 236 & 103.00 & 0.44 & $95.87 \%$ \\
Total variation & 471 & 213.78 & 0.46 & $100.0 \%$ \\
\hline
\end{tabular}

$* \mathrm{P}<0.05$. d.f. $=$ degrees of freedom.

The results in Figure 2 were obtained using an NJ clustering tree for the four wild stocks of M. nipponense in Qiandao Lake. The 09CA and 09JJ stocks formed one group, while the 09AY and 09WP stocks were clustered into another one.

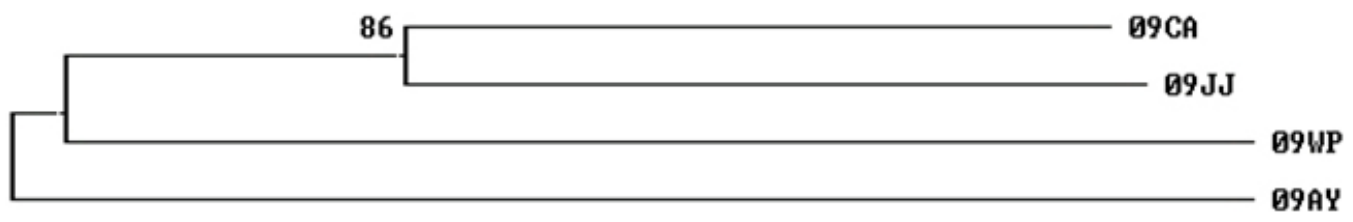

Figure 2. Neighbor-joining clustering tree based on $D_{\mathrm{A}}$ genetic distance. $09 \mathrm{CA}=$ wild stock of Chun'an town; 09AY = wild stock of Anyang town; 09JJ = wild stock of Jiangjia town; 09WP = wild stock of Weiping town.

The structure calculation does not require knowledge of the population's genetic background in advance. Therefore, the Structure software is considered as an ideal tool for population genetic structure analysis (Falush et al., 2003). After 10 repeated tests, a scatter curve was made by means of $\operatorname{Var}[\operatorname{LnP}(\mathrm{D})]$ value $[\mathrm{LnP}(\mathrm{D})=-13532.7, \mathrm{~K}=1 ; \mathrm{LnP}(\mathrm{D})=$ $-13013.3, \mathrm{~K}=2 ; \mathrm{LnP}(\mathrm{D})=-13017.1, \mathrm{~K}=3 ; \mathrm{LnP}(\mathrm{D})=-13022.4, \mathrm{~K}=4]$ (Evanno et al., 2005). When $\mathrm{K}$ equaled 2, a turning point appeared. The four stocks were divided into two potential 
populations. One population consisted of the 09CA stock, and the other three stocks belonged to another population (Figure 3).

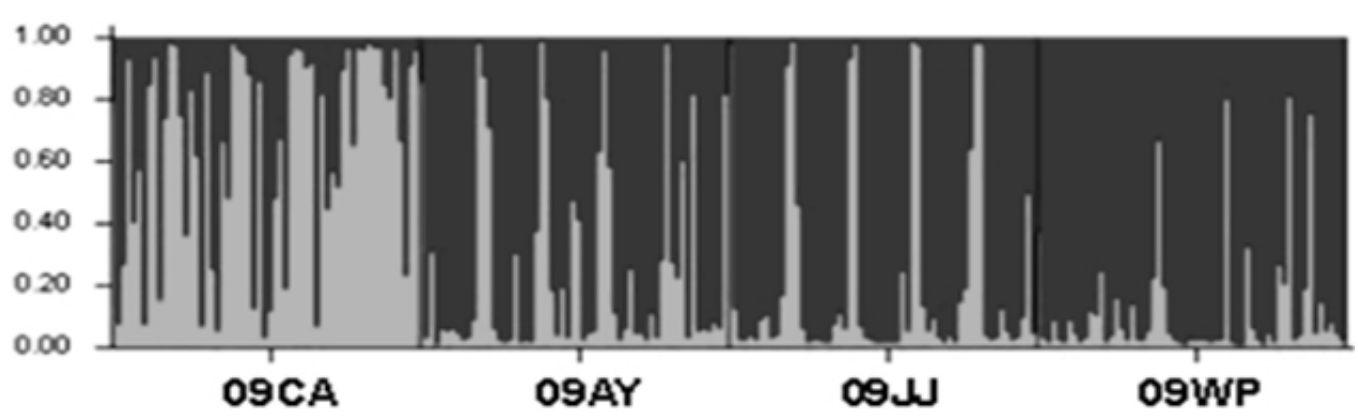

Figure 3. Two divided potential populations of Macrobrachium nipponense based on genetic structure of $\mathrm{K}$ value. 09CA = wild stock of Chun'an town; 09AY = wild stock of Anyang town; 09JJ = wild stock of Jiangjia town; $09 \mathrm{WP}=$ wild stock of Weiping town. $\mathrm{X}$-axis $=$ stock codes; $\mathrm{Y}$-axis $=$ percentage of germplasm. Each individual is represented by a single-vertical line.

\section{DISCUSSION}

Genetic variation is the basis of a selective breeding program. Generally, high genetic variation promises high genetic improvement of traits through selection, provided that the traits were determined mainly by additive genetic factors (Liu and Cordes, 2004). High genetic variation is also essential for the survival of populations because genetic variation within a certain range can allow adaptation to a changing environment. However, genetic variation is difficult to predict without polymorphic genetic markers (Liu and Cordes, 2004). The nine microsatellites used in this study were polymorphic in all four wild M. nipponense stocks. Across the four stocks, a total of 185 alleles were detected, with an average of 20 alleles/locus, suggesting that $M$. nipponense in Qiandao Lake has a high genetic variation. The high allele number observed at the nine loci allowed us to use more powerful statistical tools to analyze the stock structure (Estoup et al., 1998).

The 09CA, 09AY and 09JJ stocks showed higher genetic diversity than the 09WP stock. There are several possible explanations for this. The 09WP stock is located upstream of Qiandao Lake. The narrowness and shallow depth of the channel limits the impact of the Xin'anjiang Dam on its water storage. In addition, during the dry season or when the water is used to generate electricity, hydrological conditions would change and have a much greater impact on the 09WP stock than on the other stocks. For example, the pollution in the water could have a greater impact on 09WP than the other stocks that are located downstream of the lake because the pollution usually is diluted by the waters from the middle and lower part of the river. The 09AY and 09JJ stocks are the inner bay stocks, located midstream with abundant water. Even if the water level is reduced, changing water conditions will not be as severe thanks to the hilly and karst topography that retains plenty of water.

Almost all of the microsatellite loci of M. nipponense in Qiandao Lake deviated from Hardy-Weinberg equilibrium. This might have been due to small sample size, null alleles, or nonrandom mating of $M$. nipponense caused by changes in water flow and generation overlap. Null alleles are the result of mutated bases at flanking regions of the microsatellite sites. 
However, null alleles have been checked and corrected in our study, so this potential reason can be ruled out. Nonrandom mating may be caused by geographic isolation when water is cut off during the dry season or is used to generate electricity. The weak M. nipponense is easily preyed upon by strong $M$. nipponense individuals when environmental capacity is decreased. In addition, natural selection may be another reason for the deviation from Hardy-Weinberg equilibrium. For example, some individuals with particular alleles may die earlier under natural conditions. There was no significant departure from Hardy-Weinberg equilibrium (P > 0.05) for the Mni82 and Mni86 loci in the 09CA stock. This suggests that the 09CA stock is stable in genotype frequency and gene frequency compared to the other three stocks. The 09CA population resides in the downstream of Qiandao Lake, where a large body of water exchanges more frequently than elsewhere. Thus, the genomic stability of the 09CA stock may be due to normal gene flow caused by the natural flow of water in the downstream waters. On the other hand, the downstream of Qiandao Lake is close to the water reservoir of Xin'anjiang Dam, which makes the water environment relatively stable due to the water storage by the dam. For this reason, there is a weak environmental selection pressure on the 09CA population. However, the Xin'anjiang Dam could reduce the downstream flow, which may change the habitat of aquatic life, including M. nipponense, and this would lead to a reduction of genetic homogeneity and genetic diversity. Therefore, based on the genetic data and geographic and ecological information, nonrandom mating and natural selection may be the major reasons that M. nipponense in Qiandao Lake deviates from Hardy-Weinberg equilibrium.

Heterozygosity excess is considered a sign of populations undergoing reduction, and it can be confirmed by the infinite allele model (IAM) and minority microsatellite loci of the step-wise mutation model (SMM) (Maruyama and Fuerst, 1985; Cornuet and Luikart, 1996). TPM is an intermediate model between IAM and SMM that is composed of a large amount of one-step mutations (90-95\%) and a small amount of multi-step mutations (Luikart and Cornuet, 1998). Many microsatellite data correlate with TPM. TPM has been recommended for population bottleneck testing (Di Rienzo et al., 1994; Spencer et al., 2000). Hence, TPM is more appropriate for bottleneck analysis in this study. When a population is reduced, especially when reduced under the effective population size, it often loses rare alleles at polymorphic microsatellite loci. In our study, the two test results from mutation-drift equilibrium (Table 2) support a conclusion that there is no evidence $(\mathrm{P}>0.05)$ that the wild population of M. nipponense in Qiandao Lake has declined, suggesting that all four stocks in Qiandao Lake might not have experienced a recent bottleneck. This may be due to adequate and reasonable protection and utilization of wild $M$. nipponense in Qiandao Lake.

The $F_{\mathrm{ST}}$ value is a useful measurement of genetic differentiation among populations. In this study, the $F_{\mathrm{ST}}$ values were between 0.03 and 0.07 , and these numbers are higher than those reported for other fishery species (Xu et al., 2001; Sugama et al., 2002; Meng et al., 2009; Zhan et al., 2009). The significant genetic differentiation of M. nipponense in Qiandao Lake can be explained by environmental features, including temperature and geographical barriers (Zainudin et al., 2010). Our results show that the 09WP stock has an extremely significant differentiation with the 09CA stock and a significant differentiation with the 09AY and the 09JJ stocks. This is related to the behavior of M. nipponense; that is, M. nipponense can only move a short distance and cling to the weeds or other underwater objects most of time due to weak swimming ability (Feng et al., 2008). However, no distinct relationship between geographic and genetic distance was observed in the wild stocks of M. nipponense in Qiandao 
Lake (Figure 2). Based on the genetic variation results, $F_{\mathrm{ST}}$ values and haul distance, M. nipponense from Chun'an town and Anyang town may be used for producing hybrid vigor in a selective breeding program in Qiandao Lake.

Four stocks collected in this study can be divided into two potential populations based on genetic structure (Figure 3). One population consists of the 09CA stock, and the other population is composed of the other three stocks. Considering the geographical environment of Qiandao Lake, the 09CA stock lives in deep waters of the downstream environment; thus, the living environment is different from the upstream and midstream environments. Due to the long-term impact of Xin'anjiang Hydropower Station, hydrological characteristics, such as the water depth and flowing speed, have changed the genetic structure of M. nipponense. Consequently, it is possible to characterize the two potential populations based on the special habitat.

In summary, our results showed that the different geographic distributions and water environments of $M$. nipponense stocks can contribute to different allele numbers. Due to the impact of dam closure, the genetic diversity of wild $M$. nipponense populations in the midstream and downstream of Qiandao Lake is higher than the upstream populations. The 09CA and 09AY stocks could be used as the parental stocks for a selective breeding program in Qiandao Lake considering the parameters of genetic variations and haul distance. Measures should be taken to prevent further erosion of population genetic diversity upstream of Qiandao Lake.

\section{ACKNOWLEDGMENTS}

Research supported by the Natural Science Foundation of China (\#31001111) and the Shanghai Leading Academic Discipline Project (\#Y1101). We thank Dr. Donghong Niu for her help in data processing and Drs. Peiqiao Jia and Qigen Liu for providing the original map of Qiandao Lake.

\section{REFERENCES}

Cai YX and Shokita S (2006). Report on a collection of freshwater shrimps (Crustacea: Decapoda: Caridea) from the Philippines, with descriptions of four new species. Raffles B. Zool. 54: 245-270.

Chand V, De Bruyn M and Mather PB (2005). Microsatellite loci in the eastern form of the giant freshwater prawn (Macrobrachium rosenbergii). Mol. Ecol. Notes 5: 308-310.

Chareontawee K, Poompuang S, Na-Nakorn U and Kamonrat W (2007). Genetic diversity of hatchery stocks of giant freshwater prawn (Macrobrachium rosenbergii) in Thailand. Aquaculture 271: 121-129.

Cornuet JM and Luikart G (1996). Description and power analysis of two tests for detecting recent population bottlenecks from allele frequency data. Genetics 144: 2001-2014.

De Grave S and Ghane A (2006). The establishment of the oriental river prawn, Macrobrachium nipponense (de Haan, 1849) in Anzali Lagoon, Iran. Aquat. Invasions 1: 204-208.

Di Rienzo A, Peterson AC, Garza JC, Valdes AM, et al. (1994). Mutational processes of simple-sequence repeat loci in human populations. Proc. Natl. Acad. Sci. U. S. A. 91: 3166-3170.

Estoup A, Rousset F, Michalakis Y, Cornuet JM, et al. (1998). Comparative analysis of microsatellite and allozyme markers: a case study investigating microgeographic differentiation in brown trout (Salmo trutta). Mol. Ecol. 7: 339-353.

Evanno G, Regnaut S and Goudet J (2005). Detecting the number of clusters of individuals using the software STRUCTURE: a simulation study. Mol. Ecol. 14: 2611-2620.

Excoffier L, Laval G and Schneider S (2005). Arlequin (version 3.0): an integrated software package for population genetics data analysis. Evol. Bioinform. Online 1: 47-50.

Falush D, Stephens M and Pritchard JK (2003). Inference of population structure using multilocus genotype data: linked loci and correlated allele frequencies. Genetics 164: 1567-1587.

Feng JB and Li JL (2008). Twelve polymorphic microsatellites in oriental river prawn, Macrobrachium nipponense. Mol.

Genetics and Molecular Research 11 (4): 4235-4244 (2012)

CFUNPEC-RP www.funpecrp.com.br 
Ecol. Resour. 8: 986-988.

Feng JB, Li JL and Cheng X (2008). Research progress on germplasm resource exploitation and protection of Macrobrachium nipponense. J. Shanghai Fish. Univ. 17: 371-376.

Feng JB, Wu CL, Ding HY, Hua XM, et al. (2010). Microsatellite analysis of genetic diversity in 9 wild stocks of oriental river prawn Macrobrachium nipponense in Hongze Lake. J. Fish. Sci. Chin. 17: 218-227.

Hartl DL and Clark AG (1997). Principles of Population Genetics. Sinauer Associates, Sunderland.

Liu F, Xia JH, Bai ZY, Fu JJ, et al. (2009). High genetic diversity and substantial population differentiation in grass carp (Ctenopharyngodon idella) revealed by microsatellite analysis. Aquaculture 297: 51-56.

Liu Z and Cordes J (2004). DNA marker technologies and their applications in aquaculture genetics. Aquaculture 238: 1-37.

Luikart G and Cornuet JM (1998). Empirical evaluation of a test for identifying recently bottlenecked populations from allele frequency data. Conserv. Biol. 12: 228-237.

Ma KY, Feng JB, Li JL and Ding HY (2010). Twenty-four novel polymorphic microsatellite markers from oriental river prawn (Macrobrachium nipponense). Conserv. Genet. Resour. 2: 125-128.

Ma KY, Feng JB, Xie N, Feng XY, et al. (2011). Microsatellite analysis of genetic variation of the oriental river prawn Macrobrachium nipponense in Qiantang River. Zool. Res. 32: 363-370.

Maruyama T and Fuerst PA (1985). Population bottlenecks and nonequilibrium models in population genetics. II. Number of alleles in a small population that was formed by a recent bottleneck. Genetics 111: 675-689.

Meng XH, Wang QY, Jang IK, Liu P, et al. (2009). Genetic differentiation in seven geographic populations of the fleshy shrimp Penaeus (Fenneropenaeus) chinensis based on microsatellite DNA. Aquaculture 287: 46-51.

Ministry of Agriculture, China (2009). China Fisheries Yearbook. China Agriculture Press, Beijing.

Mirabdullaev IM and Niyazov DS (2005). Alien Decapods (Crustacea) in Uzbekistan. Abstracts of the II International Symposium Invasion of Alien Species in Holarctic. Borok, Russia.

Nei M, Tajima F and Tateno Y (1983). Accuracy of estimated phylogenetic trees from molecular data. II. Gene frequency data. J. Mol. Evol. 19: 153-170.

Rosenberg NA, Pritchard JK, Weber JL, Cann HM, et al. (2002). Genetic structure of human populations. Science 298: 2381-2385

Rousset F (2008). Genepop'007: a complete re-implementation of the Genepop software for Windows and Linux. Mol. Ecol. Resour. 8: 103-106.

Salman SD, Page TJ, Naser MD and Yasser AG (2006). The invasion of Macrobrachium nipponense (De Haan, 1849) (Caridea: Palaemonidae) into the southern Iraqi marshes. Aquat. Invasions 1: 109-115.

Sambrook J and Russell DW (2001). Molecular Cloning: A Laboratory Manual. 3rd edn. CSHL Press, New York.

Spencer CC, Neigel JE and Leberg PL (2000). Experimental evaluation of the usefulness of microsatellite DNA for detecting demographic bottlenecks. Mol. Ecol. 9: 1517-1528.

Sugama K, Benzie JAH and Ballment E (2002). Genetic variation and population structure of the giant tiger prawn, Penaeus monodon, in Indonesia. Aquaculture 205: 37-48.

Van Oosterhout C, Hutchinson WF, Wills DPM and Shipley P (2004). Micro-Checker: software for identifying and correcting genotyping errors in microsatellite data. Mol. Ecol. Notes 4: 535-538.

Wolfus GM, Garcia DK and Alcivar-Warren A (1997). Application of the microsatellite technique for analyzing genetic diversity in shrimp breeding programs. Aquaculture 152: 35-47.

Xu Z, Primavera JH, de la Pena LD, Pettit P, et al. (2001). Genetic diversity of wild and cultured black tiger shrimp (Penaeus monodon) in the Philippines using microsatellites. Aquaculture 199: 13-40.

Yu HP and Miyake S (1972). Five species of the genus Macrobrachium (Crustacea, Decapoda, Palaemonidae) from Taiwan. Оhти 3: 45-55.

Yue GH, Li Y, Lim LC and Orban L (2004). Monitoring the genetic diversity of three Asian arowana (Scleropages formosus) captive stocks using AFLP and microsatellites. Aquaculture 237: 89-102.

Yue GH, Zhu ZY, Lo LC, Wang CM, et al. (2009). Genetic variation and population structure of Asian seabass (Lates calcarifer) in the Asia-Pacific region. Aquaculture 293: 22-28.

Zainudin R, Shukor MN, Norhayati A, Md-Zain BM, et al. (2010). Genetic structure of Hylarana erythraea (Amphibia: Anura: Ranidae) from Malaysia. Zool. Stud. 49: 688-702.

Zhan A, Hu J, Hu X, Zhou Z, et al. (2009). Fine-scale population genetic structure of Zhikong scallop (Chlamys farreri): do local marine currents drive geographical differentiation? Mar. Biotechnol. 11: 223-235. 